\title{
Myristica Fragrans (MF): Potential Role as an Antioxidant and Anti-Inflammatory Agent
}

\author{
Sethi J ${ }^{1 *}$ and Dahiya $\mathbf{K}^{2}$ \\ ${ }^{1}$ Department of Physiology, India \\ 2Department of Biochemistry, India
}

*Corresponding author: Jyoti Sethi, Department of Physiology, Pt B D Sharma PGIMS

Research Article

Volume 2 Issue 2

Received Date: March 18, 2018

Published Date: March 29, 2018

Rohtak-124001, Haryana, India. Email: dr_jyotisethi@rediffmail.com

\begin{abstract}
The aim of the present study was to evaluate anti- inflammatory and antioxidant potential of Myristica fragrans (MF) in albino rabbits. Rabbits were divided into four groups of six each, depending on the diet received Group I: Normal control rabbits, Group II: (MF group), Group III: (HFD group), Group IV: (HFD with MF group). Blood samples were collected from the marginal vein of pinna of overnight fasted rabbits at the end of experimental period for estimation of Malondialdehyde (MDA), Reduced glutathione (GSH) and Vitamin C. Significant anti-inflammatory and anti-lipid peroxidative effect was observed in animals who received supplementation of Myristica fragrans along with high fat diet induced oxidative stress. Statistically significant increase in antioxidant parameters (Vit C, GSH) was observed with Myristica fragrans supplementation. The results suggest the antioxidant and anti inflammatory activity possessed by nutmeg could be helpful in preventing or slowing the progress of various oxidative stress-related diseases and inflammatory diseases.
\end{abstract}

Keywords: Myristica fragrans; Anti inflammatory; Antioxidant; Oxidative stress; Anti-lipidperoxidative

Abbreviations: MF: Myristica fragrans; NO: Nitric Oxide; MDA: Malondialdehyde; ROS: Reactive oxygen species; IAEC: Institutional Animal Ethical Committee

\section{Introduction}

Spices are defined as vegetable substances of indigenous origin which are aromatic or have a hot piquant taste, used to enhance the flavour of foods or adding stimulating ingredients to the foods [1]. They have been used in Indian cooking for thousands of years and are recognized as valuable, safe and naturally available source of medicine for treating various ailments [2]. Myristica fragrans (MF) Houtt (Myristicaceace) is a native of Molluccas, now cultivated in many tropical countries. It is commonly known as Jaiphal or nutmeg. Nutmeg is bitter, astringent, antipyretic, anti-inflammatory, deodorant, carminative, digestive, aphrodisiac, antiseptic, antihelminthic and toxic substance [3]. Concrete oil of nutmeg is used in cases of mild ringworm, chronic rheumatism, paralysis and sprains [4]. Few preliminary studies demonstrated sedative, antiulcerogenic, 


\section{Journal of Natural \& Ayurvedic Medicine}

antibacterial and antioxidant properties of various parts of the plant $[5,6]$.

Free radicals have an inherent ability to cause oxidative damage to biological macromolecules and thus have been implicated in the aetiology of various diseases such as cellular and metabolic injury, cancer, atherosclerosis, inflammation, aging, immunosuppresion, diabetes, ischemic heart disease and neurodegenerative disorder such as Alzheimer's and Parkinson's diseases [7]. Reactive oxygen species (ROS) production plays an important role in the modulation of inflammatory reactions Free radicals can cause damage by a variety of different mechanisms which include DNA damage, lipid peroxidation, protein damage, oxidation of enzymes (e.g. anti-protease) and stimulation of pro-inflammatory cytokines release and thus, their neutralization by antioxidants and radical scavengers can attenuate inflammation [8].

The deleterious effects of free radicals are kept under check by a delicate balance between rate of their production and elimination by antioxidant defence mechanism and any shift in this delicate balance will lead to cellular damage. Antioxidants are micronutrients that have gained importance in recent years due to their ability to neutralize free radicals or their actions [9]. The potential of extracts from medicinal plants to scavenge these free radicals and modulate inflammatory reactions has been demonstrated $[10,11]$. Therefore, in present scenario there is a continuous search for new and natural antioxidants from dietary plants, because of their safety, efficacy and cost effectiveness which can be useful against a variety of oxidative stress related diseases. The aim of the present study was to evaluate the effects of aqueous extract from the fruit MF to scavenge these free radicals and modulate inflammatory reactions.

\section{Materials and Methods}

\section{Animals}

Albino rabbits of either sex weighing $1.5-2.5 \mathrm{~kg}$ were used as subjects for this study. Animals were procured from disease free animal house of CCS Haryana Agriculture University, Hisar (Haryana, India). They had free access to food and water and were maintained under 12:12 hour light and dark cycles. Institutional Animal Ethical Committee (IAEC) approved the experimental protocol (No. Phy/09/413 dated 13.5.09) and care of animals was taken as per guidelines of CPCSEA, Department of Animal Welfare, Government of India.

\section{Experimental Design}

Rabbits were divided into four groups of six each, depending on the diet received.

Group I: (Control group): Received standard chow diet throughout the experiment for 14 weeks.

Group II: (MF group): Rabbits received MF fruit aqueous extract $(300 \mathrm{mg} / \mathrm{kg}, \mathrm{PO})$ daily throughout the experiment for14 weeks.

Group III: (HFD group): Rabbits received high fat diet daily throughout the experiment for 14 weeks.

Group IV: (HFD with MF group): Rabbits received high fat diet for 14 weeks and then MF fruit aqueous extract (300 $\mathrm{mg} / \mathrm{kg}$, PO) daily throughout the experiment for 4 weeks (HFD with MF group).

The composition of two diets was as follows: [12]

Control diet: Wheat flour 22.5\%, roasted Bengal gram powder $60 \%$, skimmed milk powder $5 \%$, casein $4 \%$, refined oil $4 \%$, salt mixture with starch $4 \%$ and vitamins and choline mixture $0.5 \%$.

HFD: Wheat flour 20.5\%, roasted Bengal gram powder $52.6 \%$, skimmed milk powder $5 \%$, casein $4 \%$, refined oil $4 \%$, coconut oil $9 \%$, salt mixture with starch $4 \%$, vitamins and choline mixture $0.5 \%$ and cholesterol $0.4 \%$.

Aqueous extract from powdered fruit of MF was prepared by standard procedure. Oral dose of extract was selected after a pilot study using three doses i.e. 100, 200 and $300 \mathrm{mg} / \mathrm{kg}$. All the drugs were administered orally (using an intragastric tube) in a single dose in the morning.

\section{Sample Collection}

Blood samples were collected from the marginal vein of pinna of overnight fasted rabbits at the end of the experimental period. Nitric Oxide (NO), Malondialdehyde (MDA) and antioxidant parameters- GSH (Reduced glutathione) and Vitamin $\mathrm{C}$ were determined at the end of the study. The serum was separated immediately and assayed for the following parameters.

- Nitric Oxide (NO): NO levels were estimated using the Greiss reaction. The Greiss reaction measures nitrite formed from NO, which has a short half-life of 6-10 seconds. Nitrite is a stable and non-volatile end product of NO and reacts with Greiss reagent to form a purplecolored complex whose absorbance is read at $546 \mathrm{~nm}$ [13].

- Malondialdehyde (MDA): The lipid peroxidation products reacted with thiobarbituric acid (TBA) to give a red chromogen, which was measured at 535nm, spectrophotometrically [14]. 


\section{Journal of Natural \& Ayurvedic Medicine}

- Reduced Glutathione (GSH): Deproteinised serum was precipitated with metaphosphoric acid and was made to react with 5, 5'dithiobis-2-nitrobenzoic acid (DTNB) to produce a yellow chromogen, and absorbance was measured at $420 \mathrm{~nm}$ against blank [15].

- Vitamin C: Ascorbic acid in the presence of $\mathrm{Cu}^{2+}$ was oxidized to dihydroascorbic acid which reacted with 2,4 dinitrophenylhydrazine (DNPH) to form a red bishydrazone with a maximum absorbance at $520 \mathrm{~nm}$, and thiourea was added to prevent oxidation of DNPH by any interfering substance [16].

\section{Statistical Analysis}

The results were expressed as mean \pm SEM and were analysed using student $t$ test (SPSS-14) and $\mathrm{p}$ value $<0.05$ was considered significant.

\section{Results}

In the present study there was statistically significant increase in NO levels and MDA levels (represent lipid peroxidation) in group III as compared to Group I. Oxidative stress also led to significant depletion of antioxidant defence mechanism as evidenced by decrease in levels of GSH and Vit C in group III. As shown in Table 1, administration of MF $(300 \mathrm{mg} / \mathrm{kg})$ aqueous seed extract significantly decreased NO and MDA levels simultaneously increased GSH and Vit C in Group II rabbits as compared to the control group $(\mathrm{P}<0.001)$. Supplementation of MF along with HFD decreased the NO levels and MDA levels in group IV $(\mathrm{p}<0.05)$ as compared to group III, probably through positive modulation of GSH and Vitamin C levels.

\begin{tabular}{|c|c|c|c|c|}
\hline & MDA N mol/ml & NO U mol/L & GSH U mol/ml & Vit C mg/dl \\
\hline Group-I(C) & $3.24 \pm 0.76$ & $23.6 \pm+5.67$ & $0.64 \pm 0.084$ & $1.013 \pm 051$ \\
\hline Group-II (MF) & $2.53 \pm 0.78^{*}$ & $20.9 \pm 4.82^{*}$ & $0.942 \pm 0.42^{*}$ & $1.210 \pm 0.48^{*}$ \\
\hline Group-III(HFD) & $6.22 \pm 0.70^{*}$ & $25.6 \pm 7.12^{*}$ & $0.381 \pm 0.15^{*}$ & $0.68 \pm 0.40^{*}$ \\
\hline Group-IV(HFD+MF) & $4.22 \pm 0.72^{\#}$ & $22.7 \pm-5.12^{\#}$ & $0.491 \pm 0.26^{\#}$ & $0.83 \pm 0.50^{\#}$ \\
\hline
\end{tabular}

$*:-\mathrm{p}<0.01$ as compared to group-I

\# :- $\mathrm{p}<0.05$ as compared to group-III

Table 1: Effect of aqueous Myristica fragrans extract (300 mg/ kg, p.o.) on MDA, NO and antioxidant parameters in rabbits. Data are given as mean \pm SEM $(n=6$ in each group).

\section{Discussion}

Inflammation is a normal protective response induced by tissue injury or infection to combat invaders in the body (microorganisms and non-self-cells) and to remove dead or damaged host cells [17]. In the inflammatory response there is an increase in permeability of endothelial lining cells and influx of blood leukocytes into the interstitium, oxidative burst, and release of cytokines. ROS promote inflammation by stimulating the release of the cytokines such as interleukine-I, tumor necrosis factor- $\alpha$, and interferon- $\gamma$, which stimulate recruitment of additional neutrophil and macrophages. It is well known that NO and PGE2 are main macrophage-derived inflammatory mediators [18]. Excessive production of NO could however potentially lead to tissue damage and activation of pro-inflammatory mediators [19]. Thus the use of dietary antioxidants can prevent or retard the oxidation of susceptible cellular substrates and can help in reducing the severity of inflammatory diseases. The aim of present study was to evaluate the antioxidant and anti-inflammatory role of MF. In the present study, animals of gp III (HFD) showed an increase in NO levels as well as MDA levels suggesting the role of free radicals in the pathogenesis of oxidative stress.

Gupta, et al. [20] evaluated the antioxidant effect of various nutmeg seed extracts (acetone, ethanol, methanol, butanol and water) and reported that acetone extract possessed the highest antioxidant activity. Tan, et al. [21] have reported that Nutmeg is rich in phenolic constituents, and determined the FRAP (ferric reducing antioxidant power) and TEAC (trolox equivalnt antioxidant capacity) of MF. Since It has been reported that the total phenolic content and anti-oxidant potential of a product has a significant and positive correlation [22]. So it was concluded that MF demonstrated good anti-oxidant capacity which could be attributed to presence of various phytochemicals, mainly vitamins, carotenoids, terpenoids, alkaloids, flavonoids, lignans, simple phenols and phenolic acids Jimoh, et al. [23] concluded that the total phenolics content and high DPPH radical scavenging activities of the ethylacetate and ethanol extracts of Myristica fragrans is due to the presence of tannins and flavonoid which serve as electron donor to scavenging of free reactive oxygen species . 


\section{Journal of Natural \& Ayurvedic Medicine}

These results are in accordance with the reports of Gayathri and Anuradha [24].

In the present study, MF supplementation led to significant reduction in NO levels, thus suggesting an antiinflammatory role. These results are in accordance with study of Dewi, et al. [25] in which M. fragrans seed extract and quercetin compound from $M$. fragrans dosedependently inhibited NO, TNF- $\alpha$, IL- 6 and IL-1 $\beta$ production on LPS stimulated-RAW 264.7. Hamalainen, et al. [26] have reported that quercetin can inhibit NO production and iNOS protein and mRNA expression. M. fragrans seed contains several active phytochemicals including isoeugenol, methyl-eugenol, eugenol, dihydroguaiaretic acid, $\gamma$-terpinene, terpinen-4-ol, myristic acid, oleanolic acid, palmitic acid, camphene, lauric acid, myrecne, kaempferol, and also quercetin as the most common compound found in the M. fragrans seed [27]. Quercetin, the most commonly occuring flavonoid is an excellent antioxidant that is also suggested to possess other beneficial activities [28].

Our study strongly supports the ethno-pharmacological importance of the nutmeg. The antioxidant and anti inflammatory activity possessed by nutmeg could be helpful in preventing or slowing the progress of various oxidative stress-related diseases and inflammatory diseases. The potential of extracts from medicinal plants to scavenge these free radicals and modulate inflammatory reactions has been demonstrated. Hence proper isolation of the active principles might help in the findings of new lead compounds in the fields of antioxidant and anti-inflammatory drug research.

\section{References}

1. Enabulele SA, Ehiagbonare JE (2011) Antimicrobial, nutritional and phytochemical properties of Perinari excelsa seeds. International Journal of Pharma and Bio Sciences 2(3): 459-470.

2. Bautista Cruz A, Arnaud Viñas MR, Martínez Gutiérrez GA, Sánchez-Medina PS, Pacheco RP (2011) The traditional medicinal and food uses of four plants in Oaxaca, Mexico. J Med Plants Res 5(15): 3404-3411.

3. Sharma PC, Velma MB, Dennis TJ (2005) Database on medicinal plants used in Ayurveda. (4). New Delhi: Central Council for Research in Ayurveda and Siddha, Department of ISM \& H. Ministry of Health and Family Welfare, Government of India pp: 213-41.
4. Bhagwat AW, Saifi AQ (1980) Observations on the pharmacological actions of the volatile oil of Myristica fragrans HOUTT. J Sci Res 2(3): 183-186.

5. Dhuley JN, Naik SR (1998) Protection by Rhinax in various models of rats. J Ethnopharmacol 63(3): 219225.

6. Dorman HJD (2000) In vitro evaluation of antioxidant activity of essential oils and their components. Flavour Fragr J 15(1): 12-16.

7. Halliwell B (1994) Free radicals, antioxidants, and human disease: curiosity, cause, or consequence? Lancet 344 (8924): 721-724.

8. Pendyala G, Thomas B, Kumari S (2008) The challenge of anti-oxidants to free radicals in periodontitis. J Indian Soc Periodontol 12(3): 79-83.

9. Tiwari AK (2004) Antioxidants: New-generation therapeutic base for treatment of polygenic disorders. Curr Sci 86(8): 1092-1100.

10. Lee MH, Lee JM, Jun SH, Lee SH, Kim NW, et al. (2007) The anti-inflammatory effects of Pyrolae herba extract through the inhibition of the expression of inducible nitric oxide synthase (iNOS) and NO production. J Ethnopharmacol 112(1): 49-54.

11. Lee CJ, Chen LG, Liang WL, Wanga CC (2010) Antiinflammatory effects of Punica granatum Linne in vitro and in vivo. Food Chem 118(2): 315-322.

12. Kottai Muthu A, Sethupathy S, Manavalan R, Karar PK (2006) Antioxidant potential of methanolic extract of Dolichos biflorus Linn in high fat diet fed rabbits. Indian J Pharmacol 38(2): 131-132.

13. Dimitrios T (2007) Analysis of nitrite and nitrate in biological fluids by assays based on the Griess reaction: appraisal of the Greiss reaction in the Larginine/ nitric oxide area of research. J Chromatogr B Analyt Technol Biomed Life Sci 851(1-2): 51-70.

14. Kumar R, Seth RK, Sekhon MS, Bhargava JS (1995) Serum lipid peroxide and other enzyme levels of patients suffering from thermal injury. Burns 21(2): 96-97.

15. Beuler E, Duron O, Kelly BM (1963) Improved method for determination of blood glutathione. J Lab Clin Med 61: 882-888. 


\section{Journal of Natural \& Ayurvedic Medicine}

16. McCormick DB, Greene HL (1999) Vitamins In: Burtis CA (Ed) Ashwood ER (Eds.), Teitz Textbook of Clinical Chemistry. $3^{\text {rd }}$ (Edn.), WB Saunders, USA pp: 1023 1025.

17. Medzhitov R (2008) Origin and physiological roles of inflammation. Nature 454: 428-435.

18. Soromou L, Zhang Z, Li R, Chen N, Guo W, et al. (2012) Regulation of inflammatory cytokines in lipopolysaccharide-stimulated RAW 264.7 murine macrophage by 7-0-methyl-naringenin. Molecules 17(3): 3574-3585.

19. Moncada S, Higgs E (2006) Nitric oxide and the vascular endothelium. Handb Exp Pharmacol 176(1): 21354.

20. Gupta AD, Bansal VK, Babu V, Maithil N (2013) Chemistry, antioxidant and antimicrobial potential of nutmeg (Myristica fragrans Houtt). J Gen Eng Biotech 11(1): 25-31.

21. Tan KP, Khoo HE, Azrina A (2013) Comparison of antioxidant components and antioxidant capacity in different parts of nutmeg. International Food Research Journal 20(3): 1049-1052.

22. Velioglu YS, Mazza G, Gao L, Oomah BD (1998) Antioxidant activity and total phenolics in selected fruits, vegetables and grain products. J Agric Food Chem 46(10): 4113-4117.

23. Jimoh SO, Popoola OH, Alabi KA (2017) Radical Scavenging Capacity and Efficacy of Myristica fragrans (Nutmeg) Metabolites on Cladosporum herbarum of Food Origin. Microbiol Res J Int 20(1): 18.

24. Gayathri R, Anuradha V (2015) Phytochemical screening and total phenolic content of aqueous and acetone extracts of seed, butter, mace of nutmeg (Myristica fragrans Houtt). International J Pharma Sci Rev Res 33(1): 236-239.

25. Dewi K, Widyarto B, Erawijantari PP, Widowati W (2015) In vitro study of Myristica fragrans seed (Nutmeg) ethanolic extract and quercetin compound as anti-inflammatory agent. Int J Res Med Sci 3(9): 2303-2310.

26. Hamalainen M, Nieminen R, Vuorela $P$, Heinonen $M$, Moilanen E (2007) Anti-inflammatory effects of flavonoids: genistein, kaempferol, quercetin, and daidzein inhibit STAT-1 and NF-KB Activations, whereas flavone, isohamnetin, naringenin, and pelargonidin inhibit only NF-KB activation. Mediators Inflamm 45673.

27. Suhaj M (2006) Spice antioxidants isolation and their antiradical activity: A review. J Food Compost Anal 19(6-7): 531-537.

28. Manjeet K, Ghosh B (1999) Quercetin inhibits LPS induced nitric oxide and tumor necrosis factor-alpha production in murine macrophages. Int $\mathrm{J}$ Immunopharmacol 21(7): 435-443. 